\title{
A Magnetic Alternative to the Standard Viscous Accretion Disc
}

\author{
C.G. Campbell \\ Department of Mathematics, University of Newcastle upon Tyne, NE1 \\ $7 R U, U K$
}

\begin{abstract}
A magnetic alternative to the standard viscous accretion disc is presented. The magnetic field is generated and maintained in the disc by a dynamo mechanism. Magnetic stresses dominate in the advection of angular momentum, and only weak turbulence is needed to generate the required field.
\end{abstract}

\section{Introduction}

Accretion discs play a central role in stellar astrophysics. They occur around the compact components in close binary stars, around young stellar objects in $T$ Tauri stars, and are also believed to exist in active galactic nuclei. They are usually the main luminosity source in such systems, derived from the gravitational binding energy released by infalling matter.

The fundamental problem in disc theory is to explain the angular momentum advection required to allow inflow of material to the accreting object. Standard molecular viscosity generates azimuthal forces which are far too weak to account for this advection. An anomalous viscosity must therefore be invoked and the standard disc model due to Shakura and Sunyaev (1973) uses a parameterized form of turbulent viscosity.

This paper presents a magnetic alternative to the standard viscous disc. The magnetic field is generated and maintained in the disc by a dynamo mechanism. Azimuthal field is created by shearing of poloidal field and the feedback of $B_{\phi}$ to $\mathbf{B}_{p}$ is accomplished by a turbulent $\alpha$-process. The Balbus-Hawley instability is invoked to generate turbulence with the necessary finite mean helicity. For magnetic diffusion due to buoyancy, magnetic stresses dominate viscous stresses in the advection of angular momentum. Only weak turbulence is needed to generate the required magnetic field.

\section{The Magnetic Disc Equations}

An axisymmetric, steady disc is considered around a non-magnetic accreting star of mass $M$ and radius $R$. Cylindrical polar coordinates $(\varpi, \phi, z)$ are used, with the origin at the centre of the star and the central plane corresponding to $z=0$. The external medium is taken to be a vacuum.

The structure of the magnetic field must lead to an azimuthal force on rings of disc material, for net angular momentum advection. For a vacuum exterior, 
$B_{\phi}$ vanishes on the disc surfaces and it follows from (3) that a net $B_{\varpi} B_{\phi}$ stress is necessary for magnetic angular momentum advection. A quadrupolar-type field is the most easily generated in such a disc (e.g. Campbell 1996) and has the properties

$$
\left|B_{\varpi} / B_{\phi}\right| \ll 1, \quad\left|B_{\varpi} / B_{z}\right| \sim \varpi / h \gg 1,
$$

where $h(\varpi)$ is the disc height and the first relation follows for an $\alpha \omega$-dynamo while the second is a consequence of $\nabla \cdot \mathbf{B}_{p}=0$.

The two small quantities $\left|B_{\varpi} / B_{\phi}\right|$ and $h / \varpi$ allow the fundamental equations to be reduced to (see Campbell 1992);

$$
\begin{gathered}
v_{\phi}=v_{\mathrm{K}}=\left(\frac{G M}{\varpi}\right)^{\frac{1}{2}}, \quad \frac{z}{\varpi} \frac{v_{\mathrm{K}}^{2}}{\varpi}+\frac{1}{\rho} \frac{\partial P}{\partial z}=0, \\
v_{\varpi} \frac{\partial}{\partial \varpi}\left(\varpi^{2} \Omega\right)=\frac{1}{\mu_{0} \varpi \rho} \frac{\partial}{\partial \varpi}\left(\varpi^{2} B_{\varpi} B_{\phi}\right)+\frac{\varpi}{\mu_{0} \rho} \frac{\partial}{\partial z}\left(B_{z} B_{\phi}\right), \\
\eta \frac{\partial B_{\varpi}}{\partial z}=\alpha B_{\phi}, \quad \eta \frac{\partial^{2} B_{\phi}}{\partial z^{2}}=-\varpi B_{\varpi} \frac{d \Omega_{\mathrm{K}}}{d \varpi}, \\
\frac{1}{\varpi} \frac{\partial}{\partial \varpi}\left(\varpi \rho v_{\varpi}\right)+\frac{\partial}{\partial z}\left(\rho v_{z}\right)=0,
\end{gathered}
$$

with,

$$
\eta=\frac{\xi\left|B_{\phi c}\right|}{\left(\mu_{0} \rho_{c}\right)^{\frac{1}{2}}} h, \quad \alpha=\left\{\begin{array}{ll}
\tilde{\alpha}(\varpi), & 0<z<h, \\
0, & z=0, \\
-\tilde{\alpha}(\varpi), & -h<z<0,
\end{array} \quad \text { where } \quad \tilde{\alpha}=\epsilon\left(\frac{P_{c}}{\rho_{c}}\right)^{\frac{1}{2}}\right.
$$

Equations (2a,b) and (3) are the $\varpi, z$ and $\phi$-components of the momentum equation, $(4 \mathrm{a}, \mathrm{b})$ are the poloidal and toroidal components of the induction equation, and (5) is the continuity equation. The magnetic diffusivity, $\eta$, is due to buoyancy, with $\xi<1$ due to turbulent reconnection, and a simple form is taken for the turbulent $\alpha$ function, with $\epsilon<1$. It can be shown that the ratio of the azimuthal viscous and magnetic forces is

$$
F_{\mathrm{v} \phi} / F_{\mathrm{m} \phi} \sim \epsilon^{\frac{1}{2}} \xi^{2} \ll 1
$$

so $F_{\mathrm{v} \phi}$ is ignorable in (3).

\section{Vertical Integrals}

Combining (3) and (5) and integrating vertically, using the surface conditions $B_{\phi}(\varpi, \pm h)=\rho(\varpi, \pm h)=0$, gives

$$
\frac{d}{d \varpi}\left(\frac{\dot{M}}{2 \pi} \varpi^{2} \Omega_{\mathrm{K}}+\frac{2 \varpi^{2}}{\mu_{0}} \int_{0}^{h} B_{\varpi} B_{\phi} d z\right)=0,
$$


where $\dot{M}$ is the mass transfer rate. If $\Omega$ turns over at the edge of a boundary layer of width $\delta$ near the accretor, then $B_{\varpi} B_{\phi}=0$ can be applied at $\varpi=R+\delta$ and radial integration of $(8)$ yields

$$
\int_{0}^{h} B_{\varpi} B_{\phi} d z=-\frac{\mu_{0}(G M)^{\frac{1}{2}} \dot{M}}{4 \pi \varpi^{\frac{3}{2}}}\left[1-\left(\frac{R}{\varpi}\right)^{\frac{1}{2}}\right] .
$$

A quadrupolar-type magnetic field has the surface conditions $B_{\phi}(\varpi, h)=$ $B_{\varpi}(\varpi, h)=0$, the latter following from the thin disc approximation. Equation (4a) can be used to express the vertical integral of $B_{\varpi} B_{\phi}$ in terms of $B_{\varpi}^{2}(\varpi, 0)$ and (9) then gives

$$
B_{\varpi}^{2}(\varpi, 0)=\frac{\mu_{0}(G M)^{\frac{1}{2}} \dot{M} \tilde{\alpha}}{2 \pi \varpi^{\frac{3}{2}} \eta}\left[1-\left(\frac{R}{\varpi}\right)^{\frac{1}{2}}\right] .
$$

Vertical integration of $(2 b)$ yields

$$
P_{c}=\Omega_{\mathrm{K}}^{2} \int_{0}^{h} z \rho d z
$$

The thermal problem for an optically thick disc leads to

$$
P_{c}^{\frac{15}{2}}=\frac{3 \bar{K}}{4 \sigma}\left(\frac{\Re}{\mu}\right)^{\frac{15}{2}} h F_{+} \rho_{c}^{\frac{19}{2}}, \quad \text { with } \quad P=\frac{\Re}{\mu} \rho T,
$$

where $\sigma$ is the Stefan-Boltzmann constant, $\mu$ the mean molecular weight, $\bar{K}$ a Kramer opacity constant and $F_{+}$the surface flux.

\section{The Dynamo}

For a thin disc, the azimuthal magnetic field can be expressed in the separable form $B_{\phi}=\tilde{B}_{\phi}(\varpi) f_{\phi}(z / h)$. Substitution in $(4 \mathrm{a}, \mathrm{b})$ leads to

$$
f_{\phi}=A\left(e^{-K(\zeta-1)}-2 e^{K(\zeta-1) / 2} \cos [\sqrt{3} K(\zeta-1) / 2-\pi / 3]\right),
$$

where $A$ is a constant, $\zeta=z / h$ and the eigenvalues $K$ satisfy

$$
2 \cos (\sqrt{3} K / 2)+\exp (3 K / 2)=0, \quad \text { with } \quad K^{3}=-\frac{3 \Omega_{K} h^{3} \tilde{\alpha}}{2 \eta^{2}} .
$$

\section{The Disc Solution}

Equations (6) and (10)-(14) can be used to derive the radial structure of the disc. The result is;

$$
h(\varpi)=2.3 \times 10^{6} \frac{\xi^{\frac{1}{5}}\left|K^{5}\right|^{\frac{3}{4}}}{\epsilon^{\frac{3}{20}}}\left|\frac{f_{\phi}(0)}{f_{\phi}^{\prime \prime}(0)}\right|^{\frac{1}{5}} \frac{\dot{M}_{-10}^{\frac{3}{20}}}{M_{1}^{\frac{3}{8}}} \varpi_{8}^{\frac{9}{8}} f^{\frac{3}{20}} \mathrm{~m}
$$




$$
\begin{gathered}
B_{\varpi}(\varpi)=6.6 \times 10^{-2} \frac{\epsilon^{\frac{13}{40}}|K|^{\frac{3}{8}}}{\xi^{\frac{1}{10}}}\left|\frac{f_{\phi}(0)}{f_{\phi}^{\prime \prime}(0)}\right|^{-\frac{1}{10}} M_{1}^{\frac{7}{16}} \dot{M}_{-10}^{\frac{17}{40}} \frac{f^{\frac{17}{40}}}{\varpi_{8}^{\frac{21}{16}}} \mathrm{~T} \\
B_{\phi}(\varpi)=-9.5 \times 10^{-2} \frac{|K|^{\frac{15}{8}}}{\xi^{\frac{1}{10}} \epsilon^{\frac{7}{40}}}\left|\frac{f_{\phi}(0)}{f_{\phi}^{\prime \prime}(0)}\right|^{\frac{9}{10}} M_{1}^{\frac{7}{16}} \dot{M}_{-10}^{\frac{17}{14}} \frac{f^{\frac{17}{40}}}{\varpi_{8}^{\frac{21}{16}}} \mathrm{~T}, \\
\rho(\varpi)=9.3 \times 10^{-6} \frac{\xi^{\frac{7}{5}}|K|^{\frac{21}{4}}}{\epsilon^{\frac{21}{20}}}\left|\frac{f_{\phi}(0)}{f_{\phi}^{\prime \prime}(0)}\right|^{\frac{7}{5}} M_{1}^{\frac{5}{8}} \dot{M}_{-10}^{\frac{11}{20}} \frac{f^{\frac{11}{20}}}{\varpi^{\frac{15}{8}}} \mathrm{Kg} \mathrm{m}^{-3}, \\
T(\varpi)=3.4 \times 10^{3} \frac{\xi^{\frac{9}{5}}|K|^{\frac{27}{4}}}{\epsilon^{\frac{27}{20}}}\left|\frac{f_{\phi}(0)}{f_{\phi}^{\prime \prime}(0)}\right|^{\frac{9}{5}} M_{1}^{\frac{7}{8}} \dot{M}_{-10}^{\frac{17}{20}} \frac{f^{\frac{17}{20}}}{\varpi_{8}^{\frac{21}{8}}} \mathrm{~N} \mathrm{\textrm {m } ^ { - 2 }}, \\
v_{\varpi}(\varpi)=-2.3 \times 10^{2} \frac{\epsilon^{\frac{6}{5}}}{\xi^{\frac{8}{5}}|K|^{6}}\left|\frac{f_{\phi}(0)}{f_{\phi}^{\prime \prime}(0)}\right|^{-\frac{8}{5}} \frac{\dot{M}_{-10}^{\frac{3}{10}}}{M_{1}^{\frac{1}{4}}} \frac{1}{\varpi^{\frac{1}{4}} f^{\frac{7}{10}}} \mathrm{~m} \mathrm{~s}^{-1},
\end{gathered}
$$

where $M_{1}=M / M_{\odot}, \dot{M}_{-10}=\dot{M} / 10^{-10} M_{\odot} \mathrm{yr}^{-1}, \varpi_{8}=\varpi / 10^{8} \mathrm{~m}$ and

$$
f=1-(\varpi / R)^{\frac{1}{2}} \text {. }
$$

\section{Conclusions}

The dimensionless quantity $\epsilon$ expresses the strength of the turbulence. Selfconsistent solutions are possible for $\epsilon$ as small as $10^{-4}$, corresponding to very weak turbulence. Although the radial structure is similar to the standard viscous disc, the viscous force is negligible in the present case. The eigenvalue dependence of the magnetic disc allows different states. Typical values of $B_{\phi}$ in the central plane are $\sim 100 \mathrm{G}$, these being weakly dependent on $K$. However, the central temperature increases with $|K|$. This property of the magnetic disc may be able to be related to the outburst behaviour of discs in binary stars.

\section{References}

Campbell, C.G., 1992. Geophys.Astrophys.Fluid.Dynam., 63, 197.

Campbell, C.G., 1996. Magnetohydrodynamics in Binary Stars, Kluwer Academic Publishers.

Shakura, N.I. and Sunyaev, R.A., 1973. A\&A, 24, 337. 